\title{
Chemotherapy for advanced gastric cancer: future perspective in Japan
}

\author{
Kohei Shitara ${ }^{1}$
}

Received: 21 August 2016/Accepted: 23 September 2016/Published online: 3 October 2016

(c) The International Gastric Cancer Association and The Japanese Gastric Cancer Association 2016

\begin{abstract}
Differences in clinical outcomes between advanced gastric cancer (AGC) in Asia and that in other regions have been discussed for a long time, although no major significant differences in molecular profiles have been reported. The anti-human epidermal growth factor receptor 2 antibody trastuzumab and the anti-vascular endothelial growth factor receptor 2 monoclonal antibody ramucirumab were both approved as a treatment for AGC on the basis of global phase 3 trials including Japan. In recent years, others new agents for treatment of AGC have been investigated in global or Asian studies. Randomized phase 2 trials in Japan showed a higher response rate to $S-1$ plus leucovorin and oxaliplatin than to standard S-1 plus cisplatin, which is the rationale for an ongoing phase 3 trial in Asia (SOLAR trial). A recent global phase 1 trial of the anti-programmed cell death 1 monoclonal antibody pembrolizumab showed similar efficacy results in Asian patients and non-Asian patients, which led to large global phase 2 and phase 3 studies. Although the perspective of treatment of AGC in the near future depends on the results of ongoing large clinical trials, individualized choice of treatment based on more detailed molecular information will become important.
\end{abstract}

Keywords Chemotherapy · Gastric cancer · Human epidermal growth factor receptor 2 . Immune checkpoint inhibitors - Vascular endothelial growth factor receptor 2

Kohei Shitara

kshitara@east.ncc.go.jp

1 Department of Gastroenterology and Gastrointestinal Oncology, National Cancer Center Hospital East, 6-5-1, Kashiwanoha, Kashiwa, Chiba 277-8577, Japan

\section{Introduction}

Effective cytotoxic agents for unresectable advanced gastric cancer (AGC) or recurrent gastric cancer include 5-fluorouracil (5-FU), orally administered fluoropyrimidine, platinum agents, taxanes, irinotecan, and anthracyclines. A commonly used first-line therapy is the combination of fluoropyrimidine and platinum-based chemotherapy with or without docetaxel or anthracycline, which results in a median progression-free survival (PFS) of 5-6 months and a median overall survival (OS) of 10-15 months [1-4]. The survival benefit of secondline therapy was recently confirmed in several randomized trials [5-7]. Trastuzumab, a monoclonal antibody for human epidermal growth factor receptor 2 (HER2), significantly increased the OS of HER2-positive AGC patients [8]; thus chemotherapy plus trastuzumab therapy became standard care (Table 1). Ramucirumab, an antivascular endothelial growth factor receptor 2 (VEGFR2) antibody, is proven to be effective for previously treated AGC. The addition of ramucirumab therapy to best supportive care was associated with significantly increased OS of patients after disease progression with first-line chemotherapy [9]. Ramucirumab plus paclitaxel chemotherapy compared with paclitaxel alone also significantly prolonged OS of patients after first-line treatment [10].

Even after the introduction of these new agents, the prognosis of patients with AGC remains unsatisfactory; therefore the introduction of more effective treatments is desired. In this article, urrent knowledge of molecular profiles in gastric cancer and the status of development of chemotherapy for AGC are reviewed. 


\section{Molecular profiles in gastric cancer: Asia versus the rest of the world}

Since the establishment of HER2 as a treatment target in AGC, many findings have been reported on the expression of other growth factors or receptor tyrosine kinases (RTKs). A certain percentage of patients showed overexpression of epidermal growth factor receptor (EGFR), MET as a receptor of hepatocyte growth factor, and fibroblast growth factor receptor (FGFR) [11]. Detailed molecular profiles of gastric cancer have also been reported recently or are being evaluated in large-scale international cancer genome studies. In a study by The Cancer Genome Atlas Research Network [12], four subtypes of gastric cancers were described: (1) tumors positive for EpsteinBarr virus (EBV), (2) microsatellite instability (MSI)-high tumors, (3) genomically stable tumors, and (4) tumors with chromosomal instability. The subtype of EBV-positive cancer is characterized by frequent observations in gastric corpus, recurrent PIK3CA and ARIDIA mutations, high amplification at chromosomal locus $9 \mathrm{p}$ including the programmed death ligand 1 (PD-L1) and programmed death ligand 2 (PD-L2) genes and high expression of PD-L1 and PD-L2, extreme DNA hypermethylation, etc., whereas the MSI-high subtype shows frequent mutation of multiple genes such as HER3. On the other hand, the genomically stable subtype has a few somatic copy-number alterations but involves ARIDIA and RHOA mutations or CLDN18ARHGAP gene fusions. The subtype with chromosomal instability is rich in TP53 mutations, and has relatively numerous amplifications of RTK genes. Although The Cancer Genome Atlas Research Network study did not include tumor samples from Japan, a study of 121 Japanese patients with resected gastric cancer did not demonstrate much difference regarding gene mutations, with relatively high frequencies of TP53, PIK3CA, ARIDIA, and CDHI mutations, and kinds of gene amplifications of RTKs and of those involved in the cell cycle [13]. Molecular targeting agents for particular genomic abnormalities such as MET overexpression, MET amplification or FGFR amplifications have been evaluated for AGC. Also, MSI-high tumors are very sensitive to immune check point inhibitors. Some of these studies will be discussed in following sections.

From the immunological viewpoint, non-Asian gastric cancers are reported to be associated with a significantly higher expression of several T-cell markers (CD3, CD45RO, CD8, etc.), whereas a higher expression of the immunosuppressive transcription factor FOXP3 was reported in Japanese gastric cancers [14]. On the other hand, PD-L1 expression was reported in both Western and Japanese patient cohorts $[15,16]$. Both studies showed PDL1 expression was more frequently observed in immune cells than in tumor cells. The influence of these immunological factors on the efficacies of immune checkpoint inhibitors warrants further evaluation in clinical studies.

\section{Ongoing development in the treatment of AGC}

Various new agents have been investigated in large clinical trials for AGC (Tables 1, 2).

\section{Cytotoxic chemotherapy}

Nab-paclitaxel is nanoparticle-albumin-bound paclitaxel and does not contain Cremophor ${ }^{\circledR}$ EL or ethanol. As a result, nab-paclitaxel can reduce the risk of hypersensitivity reaction and can be administered to patients who are intolerant of alcohol. The ABSOLUTE trial is a Japanese phase 3 trial to compare the efficacy and safety of 3-weekly doses or weekly doses of nab-paclitaxel and weekly doses of Cremophor-based paclitaxel in patients with AGC refractory to first-line chemotherapy consisting of fluoropyrimidines [17]. In this study, the primary end point was achieved and showed noninferiority of weekly doses of nab-paclitaxel in terms of OS (Table 1). The median OS for weekly doses of nab-paclitaxel was 11.1 months and that for Cremophor-based paclitaxel was 10.9 months (hazard ratio $0.97,97.5 \%$ confidence interval 0.76-1.23, predefined noninferiority margin 1.25 ). The frequencies of adverse events were not significantly different between weekly doses of nab-paclitaxel and weekly doses of Cremophor-based paclitaxel. In contrast, noninferiority of nabpaclitaxel administered every 3 weeks compared with Cremophor-based paclitaxel in terms of OS was not confirmed. Moreover, nab-paclitaxel administered every 3 weeks was associated with a higher frequency of febrile neutropenia or peripheral sensory neuropathy.

A randomized phase 3 trial of S-1 plus leucovorin versus S-1 plus leucovorin plus oxaliplatin (SOL) versus S-1 plus cisplatin in AGC patients showed a higher response rate in the SOL group and longer OS [18]. The phase 3 SOLAR trial comparing TAS-118 (S-1 and leucovorin) plus oxaliplatin and S-1 plus cisplatin is ongoing in Asian countries (NCT02322593). TAS-102 is a novel oral nucleoside antitumor agent containing trifluridine and tipiracil hydrochloride, which prevents the degradation of trifluridine. In a pivotal phase 3 trial for colorectal cancer, TAS-102 showed OS benefit in comparison with placebo in patients with metastatic colorectal cancer who had been treated with standard chemotherapies [19]. A Japanese phase 2 trial of TAS-102 for pretreated AGC a showed disease control rate of $65.5 \%$ by investigator assessment [20]. The median PFS was 2.9 months and the median OS was 8.7 months. The 


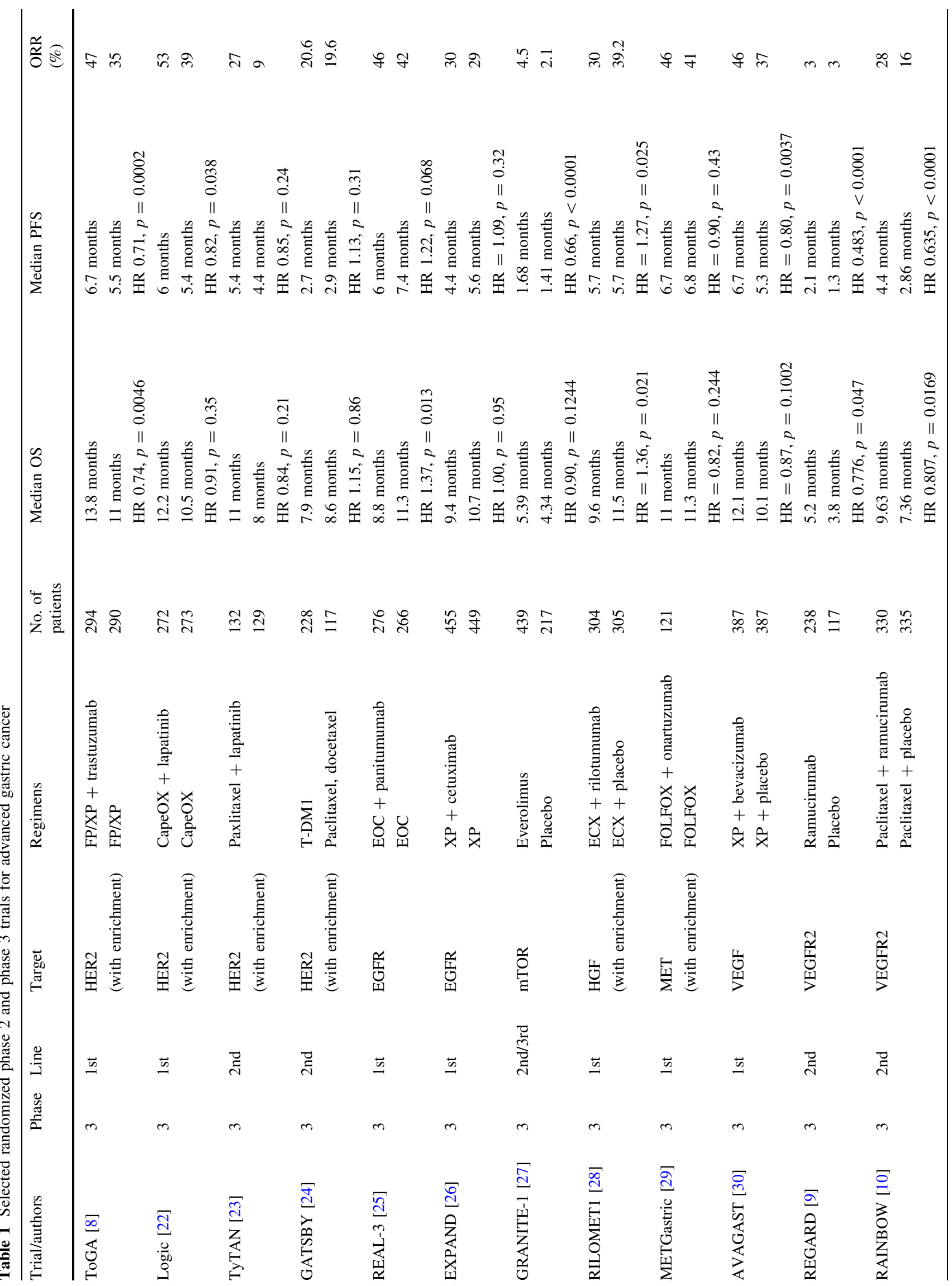




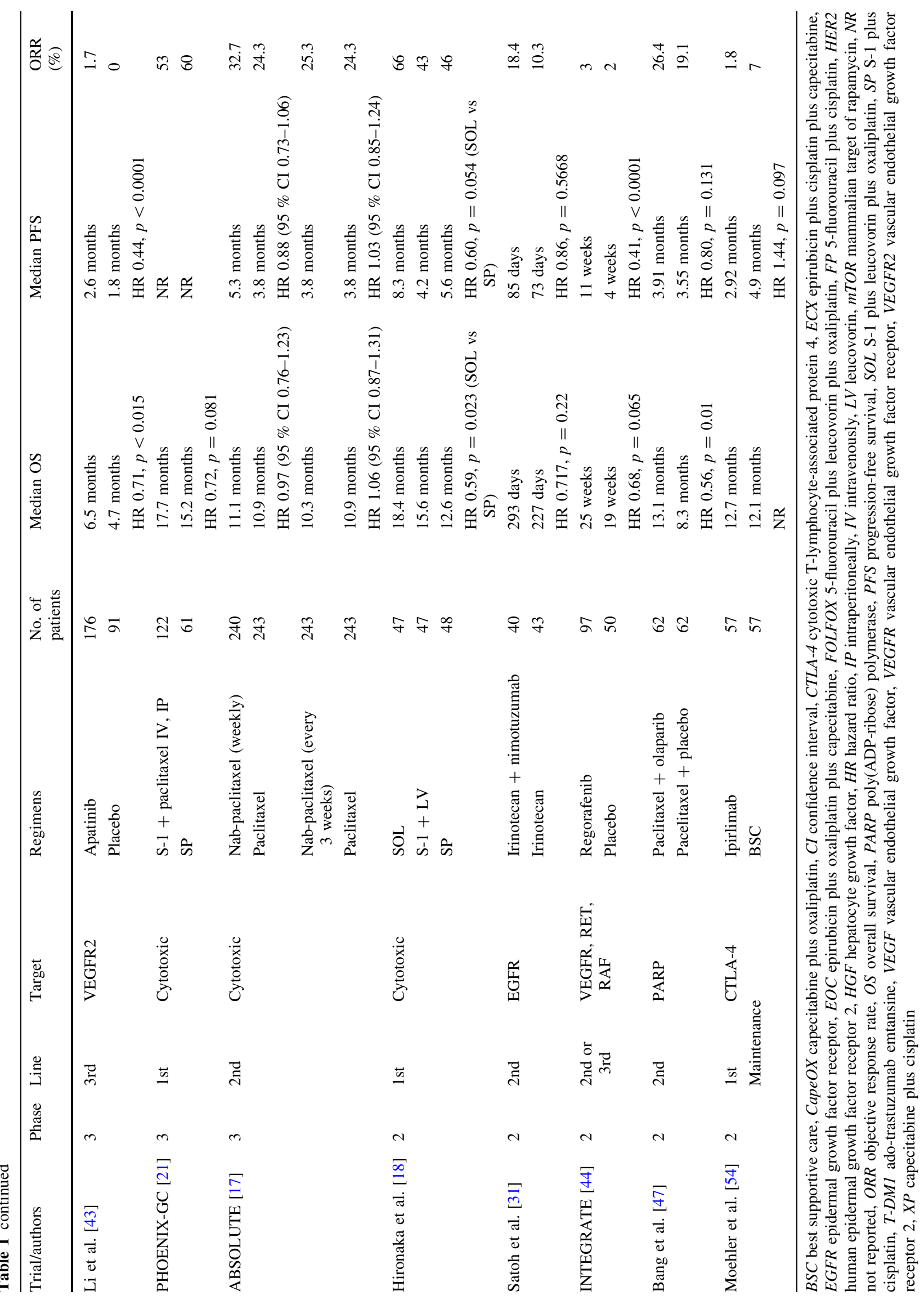


Table 2 Ongoing phase 3 trials for advanced gastric cancer

\begin{tabular}{lllll}
\hline Name of trial & Line & Control arm & Agents (experimental) & Target \\
\hline JACOB & 1st & XP or FP + trastuzumab & XP or FP + trastuzumab + pertuzumab & HER2 \\
SOLAR & $1 \mathrm{st}$ & $\mathrm{S}-1+$ cisplatin & TAS-118 + oxaliplatin & Cytotoxic \\
JCOG1013 & $1 \mathrm{st}$ & $\mathrm{S}-1+$ cisplatin & S-1 + cisplatin + docetaxel & Cytotoxic \\
RAINFALL & $1 \mathrm{st}$ & XP & XP + ramucirumab & VEGFR2 \\
KEYNOTE-062 & 1st & XP & XP + pembrolizumab & PD-1 \\
JAVELIN Gastric 100 & 1st (maintenance) & Continuation of 1st line & Avelumab & PD-L1 \\
ENRICH & 2nd & Irinotecan & Irinotecan + nimotuzumab & EGFR \\
BRIGHTER & 2nd & Paclitaxel & Paclitaxel + BBI608 & STAT3 \\
KEYNOTE-061 & 2nd & Paclitaxel & Pembrolizumab & PD-1 \\
JAVELIN Gastric 300 & 3rd & Paclitaxel/irinotecan/BSC & Avelumab & PD-L1 \\
INTEGRATE-2 (planned) & 3rd or 4th & Placebo & Regorafenib & VEGFR, RET, RAF \\
ONO-4538-12 & Last & Placebo & Nivolumab & PD-1 \\
Tags & Last & Placebo & TAS-102 & Cytotoxic \\
\hline
\end{tabular}

$B S C$ best supportive care, EGFR epidermal growth factor receptor, $F P$ 5-fluorouracil plus cisplatin, $H E R 2$ human epidermal growth factor receptor 2, $P D-1$ programmed cell death $1, P D-L 1$ programmed death ligand $1, S T A T 3$ signal transducer and activator of transcription $3, V E G F R$ vascular endothelial growth factor receptor, VEGFR2 vascular endothelial growth factor receptor 2, $X P$ capecitabine plus cisplatin

global phase 3 Tags trial is ongoing to investigate the efficacy and safety of TAS-102 in patients with AGC refractory to standard treatments (NCT02500043).

The control of peritoneal dissemination is extremely important in treatment of AGC. Intraperitoneal administration of paclitaxel provides sustained high local concentrations, and its efficacy has been shown in ovarian cancer. On the basis of previous promising results in a phase 2 trial of intraperitoneal administration of paclitaxel in combination with S-1 plus intravenous administration of paclitaxel, a phase 3 trial was conducted in Japan (PHOENIX-GC trial) [21]. A total of 183 patients were enrolled, and 164 patients were included in the efficacy analysis. The OS showed an increasing trend for intravenous regimens (median 17.7 months vs 15.2 months), although this did not reach statistical significance (Table 1). Further study might be necessary to clarify suitable candidates for intraperitoneal therapy.

\section{Molecular targeting agents}

Various drugs targeting HER2, EGFR, hepatocyte growth factor, MET, and mammalian target of rapamycin have been evaluated in AGC [22-31], but most of them did not demonstrate significant benefit in global phase 3 trials (Table 1). Even ado-trastuzumab emtansine (TDM-1), one of the antibody-drug conjugates targeting HER2 that demonstrates remarkable effectiveness in breast cancer, could not significantly prolong OS of HER2-positive AGC patients (GATSBY study) [24]. Although the exact reason for the lower efficacy in AGC than in breast cancer is not clear, it might be partially caused by the heterogeneity of
RTK expression in gastric cancers or by changes in HER2 expression after first-line treatment [32-34]. Detailed analysis of biomarkers in the GATSBY study is eagerly awaited. A phase 3 trial of pertuzumab, a monoclonal antibody for the dimerization domain of HER2, in combination of cytotoxic chemotherapy and trastuzumab therapy is ongoing (JACOB).

Large phase 3 trials of anti-EGFR monoclonal antibodies in combination with first-line chemotherapy could not show sufficient survival benefit in AGC [25, 26]. However, these two studies did not enrich patients by biomarkers. Nimotuzumab, another anti-EGFR monoclonal antibody, showed a trend of increased OS in patients with high EGFR expression [31]. A phase 3 trial in patients with EGFRpositive AGC comparing nimotuzumab plus irinotecan versus irinotecan monotherapy is ongoing (ENRICH study).

Although promising results have been obtained with MET inhibitors or FGFR inhibitors in patients with amplification of the encoding oncogenes [35-37], cases with high gene amplification rarely occur, which makes drug development challenging. A randomized phase 2 trial of AZD4547 as an FGFR inhibitor for FGFR2-amplified AGC did not show a superior outcome with this agent in comparison with paclitaxel [38]. This study enrolled patients who met the following criteria: polysomy (FISH4/5, ratio $<2.0$ and $\geq 4$ copies in $10 \%$ or more of cells), low amplification (FISH6, ratio $>2.0$ ), or high amplification (FISH6, ratio $\geq 5.0$ ) according to the $F G F R 2$ status. However, very few patients with high amplification were treated. Exploratory biomarker analysis revealed marked intratumor heterogeneity of FGFR2 amplification. In contrast, with a similar agent, three patients with very high $F G F R 2$ amplification and homogenous expression of $F G F R 2$ showed a remarkable response in 
another study [39]. Therefore the definition and the optimal cutoff of each biomarker is very important. Also, the treatment strategy to overcome intratumoral heterogeneity with use of optimal combinations warrants further evaluation in AGC.

The SCRUM project [39], a genome screening project in Japan as joint research with major domestic facilities and pharmaceutical companies, is under way, and enrollment for clinical studies targeting rare genomic alterations is anticipated. Patients with specific genomic alterations such as PIK3CA mutation, BRAF mutation, AKT1 mutation, MET or FGFR2 amplification, and FGFR3-TACC3 fusion have been enrolled in early clinical trials.

Therapy with ramucirumab, a fully human IgG1 monoclonal antibody to the extracellular vascular endothelial growth factor (VEGF)-binding domain of VEGFR2, has become one of the standard chemotherapies for pretreated AGC on the basis of the findings of two pivotal phase 3 trials (Table 1) [9, 10]. Efficacy of ramucirumab was suggested to be observed in a Japanese subgroup in the RAINBOW study [40]. Although efficacy of ramucirumab was also evaluated in a randomized phase 2 study in the first-line treatment, no increase in PFS was observed [41]. A higher rate of discontinuation of study treatment for reasons other than progressive disease in the ramucirumab arm compared with the placebo arm was observed, which led to lower study drug exposure in the experimental arm. Meanwhile, exposure-efficacy response analyses performed on data obtained from REGARD and RAINBOW demonstrated that an increase in exposure is associated with improvement in efficacy in terms of both OS and PFS [42]. The phase 3 RAINFALL trial comparing PFS for an intensive dose of ramucirumab (administration on days 1 and 8 every 21 days) with cisplatin plus capecitabine (or 5 -FU) versus placebo with cisplatin plus capecitabine (or 5-FU) as first-line treatment is ongoing (NCT02314117). Apatinib is a multikinase inhibitor which mainly targets VEGFR2 and has been developed in China. Apatinib significantly increased OS in patients with pretreated AGC [43]. Similarly, regorafenib, a multikinase inhibitor, significantly prolonged PFS compared with placebo as second-line or later-line therapy for AGC [44]. The OS showed an increasing trend with more than $50 \%$ of patients in the placebo arm receiving regorafenib as crossover treatment. A phase 3 trial is being planned.

Other drugs targeting the mechanisms of resistance to conventional chemotherapies are under development for AGC. In a preclinical study using poly(ADP-ribose) polymerase inhibitors, it was shown that these agents are effective for cell lines resistant to platinum-based agents [45]. Also, some effect has been suggested in ovarian cancer with or without BRCA mutations in clinical studies [46], leading to development in AGC patients. Promising results were obtained in a randomized phase 2 clinical study [47] which showed significant efficacy in terms of OS (Table 1). Low expression of ataxia telangiectasia mutated (ATM) protein was suggested to be associated with remarkable efficacy. However, recently it was reported by AstraZeneca that the phase 3 GOLD trial could not meet its primary end point of improving OS in all cohorts as well as the ATM-negative cohort. Detailed reports as well as biomarker analysis are awaited.

Signal transducer and activator of transcription 3 (STAT3) is a transcription factor located downstream of a variety of cytokines and Janus kinase, and it was reported that phosphorylated STAT3 activated the transcription of the Nanog and Myc genes, which are involved in the stemness of cells inside the nucleus, and that high expression of STAT3 was responsible for poor prognosis in multiple cancers [48]. BBI608 is a drug that specifically inhibits cancer stem-like cells that have CD44 positivity, high expression of STAT3, and the capability to form spheres [48]. Encouraging anticancer activity of BBI608 and paclitaxel in refractory AGC was observed in a phase $1 \mathrm{~b}$ study and a subsequent phase 2 study including 46 AGC patients, with an objective response rate (ORR) of $31 \%$ and a disease control rate of $75 \%$ [49]. The phase 3 BRIGHTER trial comparing BBI608 plus paclitaxel and placebo plus paclitaxel for AGC patients after first-line chemotherapy is ongoing.

\section{Immune checkpoint inhibitors}

Pembrolizumab is a humanized IgG4 monoclonal antibody without antibody-dependent cell-mediated cytotoxicity, and competitively inhibits the binding of programmed cell death 1 (PD-1) to PD-L1 and PD-L2. In a phase 1b gastric clinical study (KEYNOTE-012), 39 patients (19 Asians and 20 non-Asians) with PD-L1-positive AGC received pembrolizumab [50]. Immunohistochemistry with PD-L1 antibody (22C3 antibody) was performed as screening, and those with $1 \%$ or more staining in cancer cells or any staining of stromal cells were assessed as PD-L1 positive. Sixty-five of 162 patients (40 \%) were PD-L1 positive. The ORR by the central assessment was $22.2 \%$ and that of investigator judgment was $33 \%$, and a reduction in the size of the target lesions was observed in $53.1 \%$ of the patients. Although the median PFS was 1.9 months, the 6-month survival rate was $69 \%$, and the median response duration was as long as 40 weeks, with an excellent median OS of 11.4 months despite the fact that $67 \%$ of the patients had received two or more of lines of prior therapy. No significant difference in clinical outcomes was observed between Asians and non-Asians in terms of response, PFS, and OS [50]. The treatment-related adverse events were almost 
similar to those of a previous study for other solid tumors. Clinical trials of pembrolizumab are currently under way in each line of therapy, including a large phase 2 trial (KEYNOTE-059; NCT02335411), a phase 3 trial (KEYNOTE-061; NCT02370498) comparing pembrolizumab with paclitaxel as second-line treatment, and a phase 3 trial (KEYNOTE-062; NCT02494583) comparing single-agent pembrolizumab or a combination therapy of 5-FU (or capecitabine) plus cisplatin plus pembrolizumab or placebo as first-line treatment for patients with PD-L1-positive and HER2-negative AGC.

Nivolumab is a humanized IgG4 recombinant anti-PD-1 monoclonal antibody. In s phase 1/2 trial (CheckMate-032), 59 patients were treated with nivolumab monotherapy, and $83 \%$ of the patients had received two or more prior regimens [51]. The ORR was $14 \%$, the median PFS was 1.4 months, and the median OS was 5.0 months. The 6-month and 12-month survival rates were 49 and $36 \%$ respectively. A phase 3 clinical study of nivolumab in AGC patients refractory to two or more lines of treatment (ONO-4538-12) has finished recruitment and the results will be reported soon. Further, in a phase 1 trial with an expansion cohort of Japanese patients with gastric cancer, the anti-PD-L1 antibody avelumab demonstrated an ORR of $15 \%$, with a median PFS of 11.9 weeks [52]. Two phase 3 studies of maintenance therapy after first-line therapy (JAVELIN Gastric 100; NCT02625610) and third-line therapy (JAVELIN Gastric 300; NCT02625623) are ongoing. Although there have been no established biomarkers of immune checkpoint inhibitors to date, an association has been suggested, in several different types of cancers, between therapeutic effects and PD-L1 expression, types of tumorinfiltrating lymphocytes, the number of somatic mutations (mainly passenger mutation), and immune-related gene expression in tumor tissues. At this time the definitive impact of PD-L1 expression or RNA signature as biomarkers in AGC is not clear, and will be better analyzed in a cohort of larger sample size from ongoing phase 3 trials. MSI-high tumors harbor hundreds to thousands of mutations that may produce neoantigens that can be recognized and targeted by $\mathrm{T}$ cells. MSI-high colorectal cancer and noncolorectal cancers, including AGC, are very sensitive to immune checkpoint inhibitors, with an ORR of around $50 \%$ [53].

As mentioned before, single-agent response rates of immune checkpoint inhibitors are still around 10-20\%, and it is important to develop combination treatments to improve treatment outcomes. These strategies include combination of systemic chemotherapy, molecular targeting agents, radiotherapy, and local injection of oncolytic virus to enhance the local immunity and combination with immune checkpoint inhibitors such as cytotoxic T-lymphocyte-associated protein 4 (CTLA-4), lymphocyte activation gene 3 (LAG-3), and T-cell immunoglobulin domain and mucin domain 3
(TIM-3), inhibition of suppressive factors such as indoleamine 2,3-dioxygenase or transforming growth factor $\beta$, and depletion of suppressive lymphocytes such as $\mathrm{C}-\mathrm{C}$ motif chemokine receptor 4 (CCR4) antibody. Although monotherapy with the anti-CTLA-4 antibody ipilimumab did not show superior efficacy as maintenance therapy after first-line chemotherapy in comparison with best supportive care in a randomized phase 2 trial (Table 1) [54], combination of nivolumab and ipilimumab in the CheckMate-032 trial showed a relatively higher ORR than monotherapy (14\% with nivolumab monotherapy and $26 \%$ with a lower dose of nivolumab in combination with ipilimumab) [55]. A phase 3 trial of this combination is being planned. Furthermore, combination of the anti-CTLA- 4 antibody tremelimumab with the anti-PDL1 antibody MED4736 and singleagent MED4736 and tremelimub therapies are also evaluated in AGC. Regarding the combination of the existing molecular targeted drugs and immune checkpoint inhibitors, a phase 1 study of the combination of ramucirumab and pembrolizumab is ongoing [56]. VEGF-A in tumor tissues is reported to induce the expression of inhibitory molecules and to exhaust $\mathrm{CD} 8+\mathrm{T}$ cells in a mouse model, indicating the possibility of the combined use of immune checkpoint inhibitors and a VEGF inhibitor [57].

\section{Future perspectives}

Although the perspective of treatment of AGC in the near future depends on the results of ongoing clinical trials, individualized choice of treatment will become more important. Optimal timing to combine treatments and the optimal sequence of each treatment should also be evaluated in future clinical trials. Timely monitoring of tumor profiles and patients' genomic or immunological condition will be also be important to select each therapy and establish personalized therapy in AGC.

\section{Compliance with ethical standards}

Conflict of interest The authors declare that they have no conflict of interest.

\section{References}

1. Van Cutsem E, Moiseyenko VM, Tjulandin S, Majlis A, Constenla M, Boni C, et al. Phase III study of docetaxel and cisplatin plus fluorouracil compared with cisplatin and fluorouracil as firstline therapy for advanced gastric cancer: a report of the V325 Study Group. J Clin Oncol. 2006;24:4991-7.

2. Cunningham D, Starling N, Rao S, Iveson T, Nicolson M, Coxon $\mathrm{F}$, et al. Capecitabine and oxaliplatin for advanced esophagogastric cancer. N Engl J Med. 2008;358:36-46.

3. Kang YK, Kang WK, Shin DB, Chen J, Xiong J, Wang J, et al. Capecitabine/cisplatin versus 5-fluorouracil/cisplatin as first-line 
therapy in patients with advanced gastric cancer: a randomised phase III noninferiority trial. Ann Oncol. 2009;20:666-73.

4. Koizumi W, Narahara H, Hara T, Takagane A, Akiya T, Takagi M, et al. S-1 plus cisplatin versus S-1 alone for first-line treatment of advanced gastric cancer (SPIRITS trial): a phase III trial. Lancet Oncol. 2008;9:215-21.

5. Thuss-Patience PC, Kretzschmar A, Bichev D, Bichev D, Deist T, Hinke A, et al. Survival advantage for irinotecan versus best supportive care as second-line chemotherapy in gastric cancer-a randomised phase III study of the Arbeitsgemeinschaft Internistische Onkologie (AIO). Eur J Cancer. 2011;47:2306-14.

6. Kang JH, Lee SI, Lim DH, Oh SY, Kwon HC, Hwang IG, et al. Salvage chemotherapy for pretreated gastric cancer: a randomized phase III trial comparing chemotherapy plus best supportive care with best supportive care alone. J Clin Oncol. 2012;30:1513-8.

7. Ford HE, Marshall A, Bridgewater JA, Janowitz T, Coxon FY, Wadsley J, et al. Docetaxel versus active symptom control for refractory esophagogastric adenocarcinoma (COUGAR-02): an open-label, phase 3 randomised controlled trial. Lancet Oncol. 2014;15:78-86.

8. Bang YJ, Van Cutsem E, Feyereislova A, Chung HC, Shen L, Sawaki A, et al. Trastuzumab in combination with chemotherapy versus chemotherapy alone for treatment of HER2-positive advanced gastric or gastro-oesophageal junction cancer (ToGA): a phase 3, open-label, randomised controlled trial. Lancet. 2010;376:687-97.

9. Fuchs CS, Tomasek J, Yong CJ, Dumitru F, Passalacqua R, Goswami C, et al. Ramucirumab monotherapy for previously treated advanced gastric or gastro-oesophageal junction adenocarcinoma (REGARD): an international, randomised, multicentre, placebo-controlled, phase 3 trial. Lancet. 2014;383:31-9.

10. Wilke H, Muro K, Van Cutsem E, Oh SC, Bodoky G, Shimada Y, et al. Ramucirumab plus paclitaxel versus placebo plus paclitaxel in patients with previously treated advanced gastric or gastrooesophageal junction adenocarcinoma (RAINBOW): a doubleblind, randomised phase 3 trial. Lancet Oncol. 2014;15:1224-35.

11. Nagatsuma AK, Aizawa M, Kuwata T, Doi T, Ohtsu A, Fujii H, Ochiai A. Expression profiles of HER2, EGFR, MET and FGFR2 in a large cohort of patients with gastric adenocarcinoma. Gastric Cancer. 2015;18:227-38.

12. The Cancer Genome Atlas Research Network. Comprehensive molecular characterization of gastric adenocarcinoma. Nature. 2014;513:202-9.

13. Kuboki Y, Yamashita S, Niwa T, Ushijima T, Nagatsuma A, Kuwata $\mathrm{T}$, et al. Comprehensive analyses using next-generation sequencing and immunohistochemistry enable precise treatment in advanced gastric cancer. Ann Oncol. 2016;27:127-33.

14. Lin SJ, Gagnon-Bartsch JA, Tan IB, et al. Signatures of tumour immunity distinguish Asian and non-Asian gastric adenocarcinomas. Gut. 2015;64:1721-31.

15. Böger C, Behrens HM, Mathiak M, Krüger S, Kalthoff H, Röcken C. PD-L1 is an independent prognostic predictor in gastric cancer of Western patients. Oncotarget. 2016;7:24269-83.

16. Kawazoe A, Kuwata T, Kuboki Y, Shitara K, Nagatsuma A, Aizawa M, et al. Clinicopathological features of program death ligand-1 (PD-L1) expression with tumor-infiltrating lymphocytes (TILs), mismatch repair (MMR) and Epstein-Barr virus (EBV) status in a large cohort of gastric cancer. Gastric Cancer. 2016. doi:.10.1007/s10120-016-0631-3

17. Hara H, Shitara K, Takashima A, Fujitani K, Koeda K, Nakayama N, et al. Phase III trial of weekly/q3w of nab-paclitaxel vs. weekly of PTX in patients with 2nd line gastric cancer treatment. The 14th Annual Meeting of JSMO. Kobe Convention Center: Prenarysession PS1, 28-30 July 2016

18. Hironaka S, Sugimoto N, Yamaguchi K, Moriwaki T, Komatsu Y, Nishina $T$, et al. S-1 plus leucovorin versus S-1 plus leucovorin and oxaliplatin versus S-1 plus cisplatin in patients with advanced gastric cancer: a randomised, multicentre, openlabel, phase 2 trial. Lancet Oncol. 2016;17:99-108.

19. Mayer RJ, Van Cutsem E, Falcone A, Yoshino T, Garcia-Carbonero R, Mizunuma N, et al. Randomized trial of TAS-102 for refractory metastatic colorectal cancer. $N$ Engl J Med. 2015;372:1909-19.

20. Bando H, Doi T, Muro K, Yasui H, Nishina T, Yamaguchi K, et al. A multicenter phase II study of TAS-102 monotherapy in patients with pre-treated advanced gastric cancer (EPOC1201). Eur J Cancer. 2016;62:46-53.

21. Ishigami H, Fujiwara Y, Fukushima R, Nashimoto A, Yabusaki $\mathrm{H}$, Imamoto $\mathrm{H}$, et al. Phase III study of intraperitoneal paclitaxel plus s-1/paclitaxel compared with s-1/cisplatin in gastric cancer patients with peritoneal metastasis: PHOENIX-GC trial. J Clin Oncol. 2016;34(15 Suppl):4014.

22. Hecht JR, Bang YJ, Qin SK, Chung HC, Xu JM, Park JO, et al. Lapatinib in combination with capecitabine plus oxaliplatin in human epidermal growth factor receptor 2-positive advanced or metastatic gastric, esophageal, or gastroesophageal adenocarcinoma: TRIO-013/LOGiC-A randomized phase III trial. J Clin Oncol. 2016;34:443-51.

23. Satoh T, Xu RH, Chung HC, Sun GP, Doi T, Xu JM, et al. Lapatinib plus paclitaxel versus paclitaxel alone in the secondline treatment of HER2-amplified advanced gastric cancer in Asian populations: TyTAN-a randomized, phase III study. J Clin Oncol. 2014;32:2039-49.

24. Kang YK, Shah MA, Ohtsu A, Van Cutsem E, Ajani JA, van der Hors $\mathrm{T}$, et al. A randomized, open-label, multicenter, adaptive phase $2 / 3$ study of trastuzumab emtansine (T-DM1) versus a taxane (TAX) in patients (pts) with previously treated HER2positive locally advanced or metastatic gastric/gastroesophageal junction adenocarcinoma (LA/MGC/GEJC). J Clin Oncol. 2016;34(4 Suppl):5.

25. Waddell T, Chau I, Cunningham D, Gonzalez D, Okines AF, Okines $\mathrm{C}$, et al. Epirubicin, oxaliplatin, and capecitabine with or without panitumumab for patients with previously untreated advanced oesophagogastric cancer (REAL3): a randomised, open-label phase 3 trial. Lancet Oncol. 2013;14:481-9.

26. Lordick F, Kang YK, Chung HC, Salman P, Oh SC, Bodoky G, et al. Capecitabine and cisplatin with or without cetuximab for patients with previously untreated advanced gastric cancer (EXPAND): a randomised, open-label phase 3 trial. Lancet Oncol. 2013;14:490-9.

27. Ohtsu A, Ajani JA, Bai YX, Bang YJ, Chung HC, Pan HM, et al. Everolimus for previously treated advanced gastric cancer: results of the randomized, double-blind, phase III GRANITE-1 study. J Clin Oncol. 2013;31:3935-43.

28. Cunningham D, Tebbutt NC, Davidenko I, Murad AM, Al-Batran $\mathrm{SE}$, Ilson DH, et al. Phase III, randomized, double-blind, multicenter, placebo $(\mathrm{P})$-controlled trial of rilotumumab $(\mathrm{R})$ plus epirubicin, cisplatin and capecitabine (ECX) as first-line therapy in patients (pts) with advanced MET-positive (pos) gastric or gastroesophageal junction (G/GEJ) cancer: RILOMET-1 study. J Clin Oncol. 2015;33(15 Suppl):4000.

29. Shah MA, Bang YJ, Lordick F, Tabernero J, Chen M, Hack SP, et al. METGastric: a phase III study of onartuzumab plus mFOLFOX6 in patients with metastatic HER2-negative (HER2-) and MET-positive (MET+) adenocarcinoma of the stomach or gastroesophageal junction (GEC). J Clin Oncol. 2015;33(15 Suppl):4012.

30. Ohtsu A, Shah MA, Van Cutsem E, Rha SY, Sawaki A, Park SR, et al. Bevacizumab in combination with chemotherapy as firstline therapy in advanced gastric cancer: a randomized, doubleblind, placebo-controlled phase III study. J Clin Oncol. 2011;29:3968-76. 
31. Satoh T, Lee KH, Rha SY, Sasaki Y, Park SH, Komatsu Y, et al. Randomized phase II trial of nimotuzumab plus irinotecan versus irinotecan alone as second-line therapy for patients with advanced gastric cancer. Gastric Cancer. 2015;18:824-32.

32. Treacy AD, Karamchandani JR, Streutker CJ, Grin A. HER2 genetic heterogeneity in gastric cancer: evaluation according to the College of American Pathologists Breast Cancer Criteria. Appl Immunohistochem Mol Morphol. 2015;23:628-32.

33. Janjigian YY, Riches JC, Ku GY, Imtiaz T, Capanu M, Chou JF, et al. Loss of human epidermal growth factor receptor 2 (HER2) expression in HER2-overexpressing esophagogastric (EG) tumors treated with trastuzumab. J Clin Oncol. 2015;33(3 Suppl 3):63.

34. Ogitani Y, Aida T, Hagihara K, Yamaguchi J, Ishii C, Harada N, et al. DS-8201a, a novel HER2-targeting ADC with a novel DNA topoisomerase I inhibitor, demonstrates a promising anti-tumor efficacy with differentiation from T-DM1. Clin Cancer Res. 2016. doi:10.1158/1078-0432.CCR-15-2822.

35. Kwak EL, LoRusso P, Hamid O, Janku F, Kittaneh M, Catenacciet DVT, et al. Clinical activity of AMG 337, an oral MET kinase inhibitor, in adult patients (pts) with MET-amplified gastroesophageal junction (GEJ), gastric (G), or esophageal (E) cancer. J Clin Oncol. 2015;33(3 Suppl 3):1.

36. Shitara K, Oh DY, Yokota T, TW, Doi T, Hamauchi S, et al. Abstract A167: a phase I study of MET TKI SAR125844 in Asian patients (pts) with advanced solid tumors. Mol Cancer Ther 2015;14(12 Suppl 2):A167.

37. Smyth EC, Turner NC, Peckitt C, Pearson A, Brown G, Chua S, et al. Phase II multicenter proof of concept study of AZD4547 in FGFR amplified tumours. J Clin Oncol. 2015;33(15 Suppl):2508.

38. Bang YJ, Van Cutsem E, Mansoor W, Petty RD, Chao Y, Cunningham $\mathrm{D}$, et al. A randomized, open-label phase II study of AZD4547 (AZD) versus paclitaxel in previously treated patients with advanced gastric cancer (AGC) with fibroblast growth factor receptor 2 (FGFR2) polysomy or gene amplification: SHINE study. J Clin Oncol. 2015;33(15 Suppl):4014.

39. Shitara K, Miki I, Sudo T, Fujii S, Kuwata T, Okamoto W, et al. The nationwide cancer genome screening projects for gastrointestinal cancer in Japan (SCRUM-Japan GI-SCREEN): efficient identification of actionable cancer genome alterations in advanced colorectal and non-colorectal gastrointestinal cancer (GI Screen 2013-01-CRC and 2015-01-Non CRC). J Clin Oncol. 2015;33(15 Suppl):TPS4134.

40. Shitara K, Muro K, Shimada Y, Hironaka S, Sugimoto N, Komatsu Y, et al. Subgroup analyses of the safety and efficacy of ramucirumab in Japanese and Western patients in RAINBOW: a randomized clinical trial in second-line treatment of gastric cancer. Gastric Cancer. 2016;19:927-38.

41. Yoon HH, Bendell JC, Braiteh FC, Braiteh FS, Firdaus I, Philip PA, et al. Ramucirumab (RAM) plus FOLFOX as front-line therapy $(\mathrm{Rx})$ for advanced gastric or esophageal adenocarcinoma (GE-AC): randomized, double-blind, multicenter phase 2 trial. J Clin Oncol. 2014;32(15 Suppl):4004.

42. Tabernero J, Ohtsu A, Muro K, Van Cutsem E, Oh SC, Bodoky $\mathrm{G}$, et al. Exposure-response (E-R) relationship of ramucirumab (RAM) from two global, randomized, double-blind, phase 3 studies of patients (Pts) with advanced second-line gastric cancer. J Clin Oncol. 2015;33(3 Suppl 3):121.

43. Li J, Qin S, Xu J, Xiong J, Wu C, Bai Y, et al. Randomized, double-blind, placebo-controlled phase III trial of apatinib in patients with chemotherapy-refractory advanced or metastatic adenocarcinoma of the stomach or gastroesophageal junction. J Clin Oncol. 2016;34:1448-54.

44. Pavlakis N, Sjoquist KM, Tsobanis E, Martin AJ, Kang YK, Bang YJ, et al. INTEGRATE: a randomized, phase II, double-blind, placebo-controlled study of regorafenib in refractory advanced oesophagogastric cancer (AOGC): a study by the Australasian Gastrointestinal Trials Group (AGITG) - final overall and subgroup results. J Clin Oncol. 2015;33(15 Suppl):4003.

45. Michels J, Vitale I, Galluzzi L, Adam J, Olaussen KA, Kepp O, et al. Cisplatin resistance associated with PARP hyperactivation. Cancer Res. 2013;73:2271-80.

46. Ledermann J, Harter P, Gourley C, Friedlander M, Vergote I, Rustin G, et al. Olaparib maintenance therapy in patients with platinum-sensitive relapsed serous ovarian cancer: a preplanned retrospective analysis of outcomes by BRCA status in a randomised phase 2 trial. Lancet Oncol. 2014;15:852-61.

47. Bang YJ, Im SA, Lee KW, Cho JY, Song EK, Lee KH, et al. Randomized, double-blind phase II trial with prospective classification by ATM protein level to evaluate the efficacy and tolerability of olaparib plus paclitaxel in patients with recurrent or metastatic gastric cancer. J Clin Oncol. 2015;33:3858-65.

48. Li Y, Rogoff HA, Keates S, Gao Y, Murikipudi S, Mikule K, et al. Suppression of cancer relapse and metastasis by inhibiting cancer stemness. Proc Natl Acad Sci U S A. 2015;112:1839-44.

49. Becerra C, Stephenson J, Jonker DJ, Cohn AL, Asmis TR, Bekaii-Saab TS, et al. Phase Ib/II study of cancer stem cell (CSC) inhibitor BBI608 combined with paclitaxel in advanced gastric and gastroesophageal junction (GEJ) adenocarcinoma. J Clin Oncol. 2015;33(15 Suppl):4069.

50. Muro K, Chung HC, Shankaran V, Geva R, Catenacci D, Gupta $\mathrm{S}$, et al. Pembrolizumab for patients with PD-L1-positive advanced gastric cancer (KEYNOTE-012): a multicentre, openlabel, phase 1b trial. Lancet Oncol. 2016. doi:10.1016/S14702045(16)00175-3.

51. Le DT, Bendell JC, Calvo E, Kim JW, Ascierto AP, Sharma P, et al. Safety and activity of nivolumab monotherapy in advanced and metastatic $(\mathrm{A} / \mathrm{M})$ gastric or gastroesophageal junction cancer (GC/GEC): results from the CheckMate-032 study. J Clin Oncol. 2016;34(4 Suppl):6.

52. Yamada Y, Nishina T, Iwasa S, Shitara K, Muro K, Esaki T, et al. A phase I dose expansion trial of avelumab (MSB0010718C), an anti-PD-L1 antibody, in Japanese patients with advanced gastric cancer. J Clin Oncol. 2015;33(15 Suppl):4047.

53. Diaz LA, Uram JN, Wang H, Bartlett B, Kemberling H, Eyring A, et al. Programmed death-1 blockade in mismatch repair deficient cancer independent of tumor histology. J Clin Oncol. 2016; 34(15 Suppl):3003.

54. Moehler MH, Cho JY, Kim YH, Kim JW, Bartolomeo MD, Ajani $\mathrm{JA}$, et al. A randomized, open-label, two-arm phase II trial comparing the efficacy of sequential ipilimumab (ipi) versus best supportive care (BSC) following first-line (1L) chemotherapy in patients with unresectable, locally advanced/metastatic (A/M) gastric or gastro-esophageal junction (G/GEJ) cancer. J Clin Oncol. 2016;34(15 Suppl):4011.

55. Janjigian YY, Bendell JC, Calvo E, Kim JW, Ascierto PA, Sharma P, et al. CheckMate-032: Phase I/II, open-label study of safety and activity of nivolumab (nivo) alone or with ipilimumab (ipi) in advanced and metastatic (A/M) gastric cancer (GC). J Clin Oncol. 2016;34(15 Suppl):4010.

56. Herbst RS, Bendell JC, Isambert N, Calvo E, Santana-Davila R, Cassier $\mathrm{P}$, et al. A phase 1 study of ramucirumab (R) plus pembrolizumab $(\mathrm{P})$ in patients (pts) with advanced gastric or gastroesophageal junction (G/GEJ) adenocarcinoma, non-small cell lung cancer (NSCLC), or urothelial carcinoma (UC): phase 1a results. J Clin Oncol. 2016; 34(15 Suppl):3056.

57. Voron T, Colussi O, Marcheteau E, Pernot S, Nizard M, Pointet $\mathrm{AL}$, et al. VEGF-A modulates expression of inhibitory checkpoints on $\mathrm{CD} 8+\mathrm{T}$ cells in tumors. $\mathrm{J}$ Exp Med. 2015;9(212):139-48. 